\title{
Systematic review and meta-analysis of chronic kidney disease as predictor of atrial fibrillation recurrence following catheter ablation
}

\author{
Min $\mathrm{Li}^{1 *}$, Tong $\mathrm{Liu}^{2 *}$, Di Luo ${ }^{1}$, Guangping $\mathrm{Li}^{2}$ \\ ${ }^{1}$ Tianjin Medical University, Tianjin, People's Republic of China \\ ${ }^{2}$ Department of Cardiology, Tianjin Institute of Cardiology, \\ Second Hospital of Tianjin Medical University, Tianjin, People's Republic of China
}

\begin{abstract}
Background: Recent observational studies have shown that patients with chronic kidney disease (CKD) have higher risk of atrial fibrillation $(A F)$ recurrence and, therefore, the value of catheter ablation therapy in patients with CKD has been doubted. The purpose of this meta-analysis was to systematically analyze the effect of $C K D$ on recurrence of $A F$ following catheter ablation.

Methods: PubMed and Cochrane clinical trials databases were searched until August 2012. Of the 1966 initially identified studies, 4 observational studies with 1379 patients were analyzed.

Results: The meta-analysis of these studies showed that CKD was associated with higher AF recurrence rate following single catheter ablation $(H R=1.96,95 \% C I 1.35-2.85, p=0.0004)$ while there were significant differences between individual trials $\left(p=0.07\right.$ and $\left.I^{2}=58 \%\right)$. Sensitivity analysis suggested that this outcome was stable. A subgroup analysis showed that $C K D$ has higher recurrent risk in patients with 100\% paroxysmal $A F(H R=2.45,95 \%$ CI $1.28-4.70, p=0.007)$ than in patients with non $100 \%$ paroxysmal $A F(H R=1.65,95 \% C I$ $1.15-2.36, p=0.006$ ).

Conclusions: CKD was associated with higher AF recurrence rate following single catheter ablation. In addition, patients with $100 \%$ paroxysmal AF have higher risk than patients with non $100 \%$ paroxysmal AF that merits special consideration when evaluating patients for catheter-based AF ablation. Given that the CKD prevalence is rapidly increasing, there is an imperative need for better risk stratification of catheter ablation candidates. (Cardiol J 2014; 21, 1: 89-95)
\end{abstract}

Key words: chronic kidney disease, hemodialysis, atrial fibrillation, catheter ablation

\section{Introduction}

Renal function is known to be a causal reason of cardiovascular mortality and an independent predictor of a major complication of contemporary catheter ablation [1, 2]. Arrhythmia issues arise frequently in the renal disease population, spanning the spectrum from benign ectopy to sustained arrhythmias [3]. The presence of chronic kidney disease (CKD) increased the risk of atrial fibrillation (AF) recurrence after electrical cardioversion by catheter ablation $[4,5]$. Multifactorial physio-

\footnotetext{
Address for correspondence: Guangping Li, MD, PhD, Department of Cardiology, Tianjin Institute of Cardiology, Second Hospital of Tianjin Medical University, Tianjin 300211, People's Republic of China, tel: +86-22-88328368, fax: +86-22-28261158, e-mail: gp_limail@yahoo.com.cn

Received: 07.04.2013 Accepted: 03.08.2013

*The first 2 authors contributed equally to this study.
} 
logic factors, due to CKD, may take part in higher prevalence of recurrent $\mathrm{AF}$ in patients with CKD than in those without it [4]. In this comprehensive meta-analysis and systematic review we sought to examine the current evidence and investigate whether the presence of CKD increases the risk of $\mathrm{AF}$ recurrence following single catheter ablation.

\section{Methods}

We performed this analysis according to the guidelines of the Meta-analysis of Observational Studies in Epidemiology Group (MOOSE) [6].

\section{Inclusion criteria}

We included prospective or retrospective observational studies with a primary objective to analyze the association between CKD and recurrence of $\mathrm{AF}$ after catheter ablation. Titles and abstracts of all articles were evaluated and rejected following the inclusion criteria: 1) human subjects with AF; 2) renal function evaluated; 3) retrospective/prospective cohort studies; 4) baseline data available; 5) follow-up interval longer than 1 year; 6) $\mathrm{AF}$ recurrence following a single catheter ablation for CKD and non-CKD patients mentioned; 7) single catheter ablation for AF performed; $8)$ enough sample size $(n>100)$ of patients with and without CKD.

\section{Search strategies}

We carefully searched on-line database of PubMed until August 2012, and the Cochrane Controlled Trials Register Databases until August 2012 to identify relevant studies. We used the following key words: "atrial fibrillation", "chronic kidney disease", "catheter ablation”, "renal failure", "renal function", "renal insufficiency", "end-stage renal disease" and "dialysis". Titles and abstracts, as well as the reference lists of all the identified reports, were examined independently by 2 reviewers (ML and TL) in order to include potentially relevant studies. The 2 reviewers agreed on the inclusion/ /exclusion status in $90 \%$ of the reviewed studies. Disagreements were resolved by discussion or consensus of a third reviewer (GL). There was no language restriction when we included the studies. Additionally, a manual search was conducted using review articles on this topic, bibliographies of original papers, and abstracts of the scientific sessions of the American College of Cardiology, the American Heart Association, the European Society of Cardiology, and Heart Rhythm Society during the past 3 years.

\section{Quality assessment}

To limit heterogeneity secondary to differences between study designs, the quality of each study was evaluated according to the guidelines developed by the United States Preventive Task Force [7] and the Evidence-Based Medicine Working Group [8]. A point score system was applied based on the quality of the study. The following characteristics were assessed: 1) clear description of inclusion and exclusion criteria; 2 ) study sample representative for mentioned population; 3 ) clear description of sample selection; 4) full specification of clinical and demographic variables; 5) follow-up interval longer than 1 year; 6 ) no loss of follow-up; 7) clear definition of CKD; 8) clear definition of outcomes and outcome assessment; 9) temporality (assessment of CKD done before catheter ablation); 10) adjustment of possible confounders in multivariate analysis. If a study did not clearly mention one of these key points, we considered that it had not been performed. Therefore, the possibility of underestimation of the reported characteristics may be present.

\section{Data extraction}

Two blind investigators (ML and TL) independently performed data extraction using a standard data extraction form to determine eligibility for inclusion. The following information collected was tabulated: 1) publication details: first author's last name, publication year; 2) characteristics of included studies: study population, cohort design, method of AF detection, ablation strategy, definition of CKD, follow-up period, blanking period and risk estimate; 3 ) baseline data of the studied population: sample size, age, gender, body mass index, paroxysmal atrial fibrillation (PAF, \%), duration of $\mathrm{AF}$, number of patients with $\mathrm{CKD}$, etc.

\section{Statistical analysis}

The magnitude of association between CKD and recurrence of $\mathrm{AF}$ following single catheter ablation was measured by adjusted hazard ratio (HR) or relative risk (RR) with $95 \%$ confidence intervals (CI). One study [9] gave a value of $\mathrm{HR}$ by univariate analysis. Two studies [4, 10] employed Cox proportional hazard models to draw a value of adjusted HRs. However, in one study [5] neither a value of HR nor adjusted HR is available. For the present analyses, we assumed RR to be a valid approximation of HR. Log HR was transformed by taking their logarithms and standard errors were calculated from Log HR and corresponding $95 \%$ CI. We used the inverse variance method to 


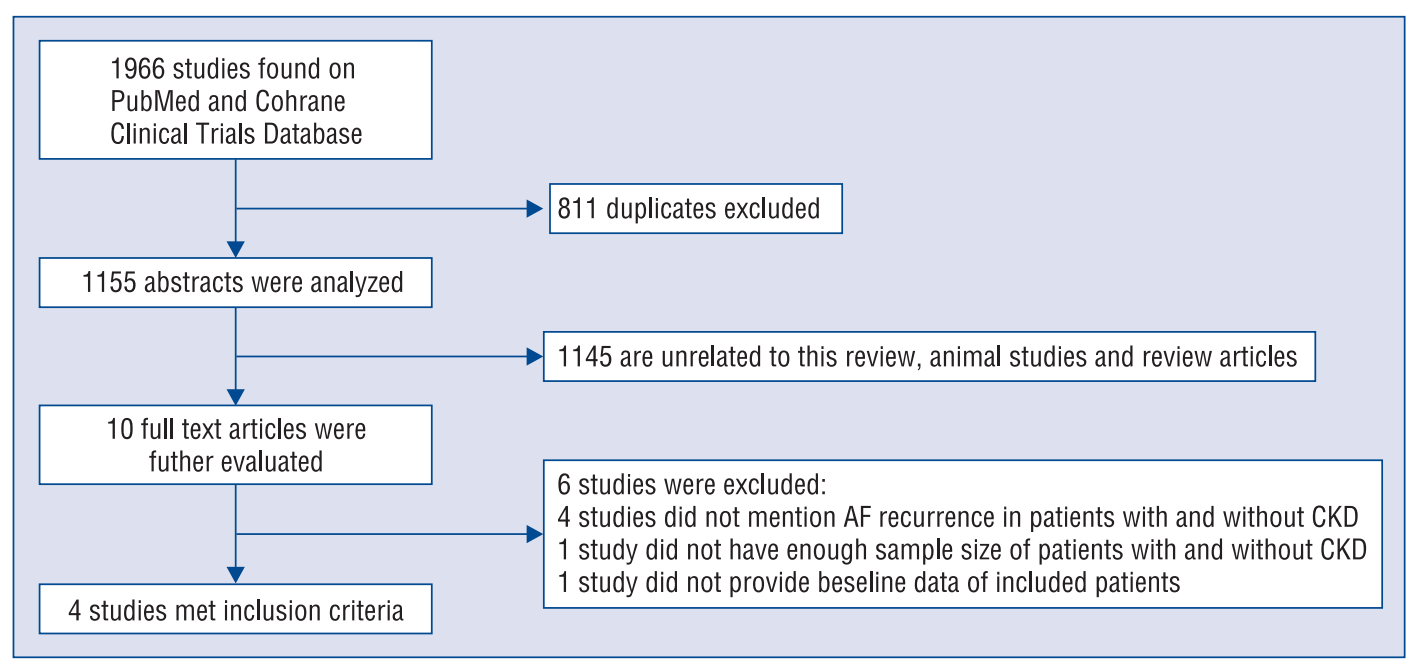

Figure 1. Flow diagram of study selection process; AF — atrial fibrillation; CKD — chronic kidney disease.

weigh studies for the combined overall statistic. Heterogeneity was examined using the standard $\chi^{2}$ test of heterogeneity. An $\mathrm{I}^{2}>50 \%$ indicates at least moderate statistical heterogeneity [11]. If the $\chi^{2}$-test for heterogeneity was significant, a pooled effect was calculated with a random-effects model which was used to take into account within-study and between-study variance, otherwise, with a fixed-effects model. Sensitivity analysis was done by leaving out studies and checking the consistency of the overall effect estimate. Statistical significance for treatment effect was defined at $\mathrm{p}$ values $<0.05$. We also performed subgroup analysis on two subsets of studies which all subjects had PAF or not. Publication bias was evaluated using funnel plot although relative small number of studies enrolled. All analyses were performed using Review Manager Version 5.1 (Revman; The Cochrane Collaboration, Oxford, UK).

\section{Results}

A total of 1966 records were identified by the primary literature search. We identified 811 duplicate articles which were discarded. However, after screening the titles and abstracts, 1155 studies were excluded because they were either laboratory studies, review articles, or irrelevant to the current analysis. Of the 10 reports selected for detailed evaluation, 6 studies did not meet the inclusion criteria and were excluded [1, 2, 12-15]. Among them, 4 [2, 12-14] did not mention AF recurrence after a single catheter ablation for CKD and non-CKD patients, 1 [1] baseline data unavailable and 1 [15] did not have enough sample size of patients with and without CKD. Consequently, $4[4,5,9,10]$ observational studies were finally included in our meta-analysis (Fig. 1). All of the 4 studies used retrospective data. There were overall 1379 patients involved in our analysis. The follow-up period ranged from 12 and 56 months. The characteristics of included studies are depicted in Table 1. The baseline data of the patients in each study are presented in Table 2 . The definitions of CKD varied: 4 studies used reduced estimated glomerular filtration rate (eGFR) assessed by the MDRD formula [16] or the Cockcroft-Gault formula.

The meta-analysis of these studies demonstrated that CKD was associated with higher recurrence rate of $\mathrm{AF}$ after catheter ablation $(\mathrm{HR}=$ $=1.96,95 \%$ CI $1.35-2.85, \mathrm{p}=0.0004$ ) (Fig. 2). The heterogeneity test showed there were significant differences between individual studies $(\mathrm{p}=0.07$, $\mathrm{I}^{2}=58 \%$ ). Sensitivity analysis was performed to find the origin of heterogeneity. After removing the study by Chao et al. [5], whose blanking period was 2 months, there was no significant heterogeneity across the studies $\left(\mathrm{p}=0.42, \mathrm{I}^{2}=0 \%\right)$. However, the overall outcome remained the same $(\mathrm{HR}=1.61$, 95\% CI 1.26-2.05, $\mathrm{p}=0.0001)$. Excluding the study by Berkowitsch et al. [10], whose definition of CKD is eGFR $<68 \mathrm{~mL} / \mathrm{min} / 1.73 \mathrm{~m}^{2}$, also reduced the heterogeneity $\left(\mathrm{p}=0.29, \mathrm{I}^{2}=20 \%\right)$ without changing the overall effect ( $\mathrm{HR}=2.32,95 \%$ CI $1.61-3.35$, $\mathrm{p}<0.00001)$. We also performed a subgroup analysis (Fig. 3) which demonstrated that CKD was associated with higher recurrent risk in patients with $100 \%$ PAF $(\mathrm{HR}=2.45,95 \%$ CI $1.28-4.70$, 


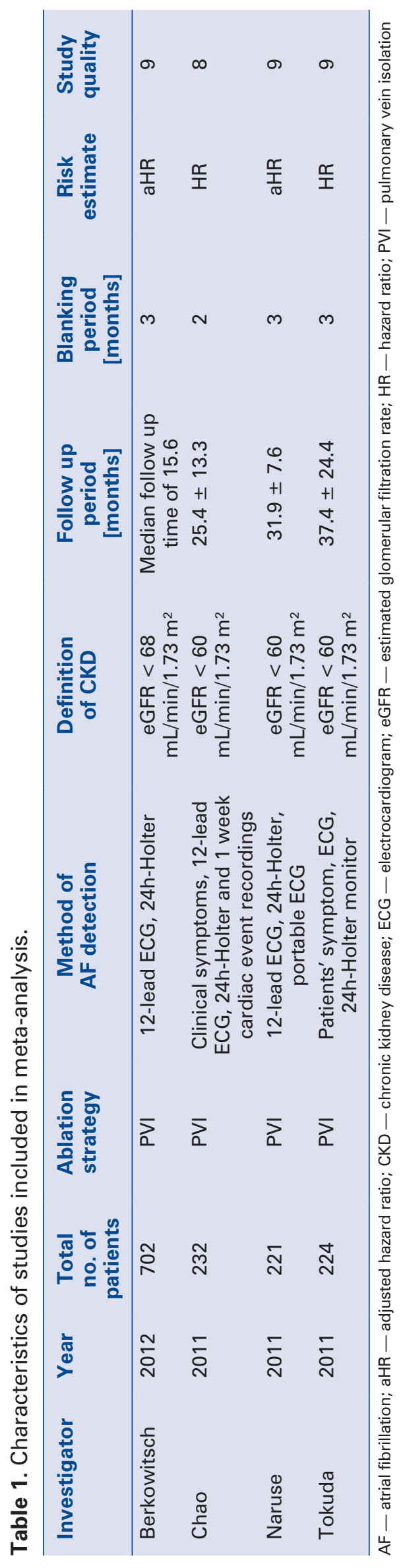

$\mathrm{p}=0.007)[5,9]$ than in patients with non $100 \%$ PAF $(\mathrm{HR}=1.65,95 \%$ CI $1.15-2.36, \mathrm{p}=0.006)$ $[4,10]$. The funnel plot (Fig. 4) suggested that there was no publication bias, although the small number of studies made it difficult to interpret.

\section{Discussion}

In this comprehensive meta-analysis, we showed that patients with CKD were associated with increased rate of $\mathrm{AF}$ recurrence following single catheter ablation. The prevalence of AF is much greater among persons with end-stage renal disease than among the general population [17]. Recent studies indicated that CKD and a decreased GFR was associated with high recurrence rate of catheter ablation in patients with PAF $[4,5,9]$. Another study clearly demonstrated that the outcome after $1^{\text {st }}$ catheter ablation of $\mathrm{AF}$ is poor in patients with impaired renal function [10]. A significant elevation in $\mathrm{AF}$ risk was observed at a threshold eGFR of $<60 \mathrm{~mL} / \mathrm{min} / 1.73 \mathrm{~m}^{2}$ [18].

Cardiorenal syndrome leads to higher numbers of individuals suffering from bidirectional disorder [19]. Regardless of severity, CKD is associated with an increased prevalence of AF among US adults [20]. In a large population-based study, reduced kidney function was strongly associated with the incidence of AF independently of other risk factors [21]. Elimination of AF by catheter ablation improved kidney function in patients with mild to moderate kidney dysfunction [1].

Several pathophysiologic mechanisms have been proposed as being responsible for the association between $\mathrm{CKD}$ and $\mathrm{AF}$. CKD may cause cardiac and atrial structural changes which are responsible for the increased incidence of cardiac arrhythmias [22]. Metabolic abnormalities such as metabolic acidosis, dyskaliemia and dyscalcemia can also lead to an increased risk of AF [23]. Accumulated evidence suggests oxidative stress may play an important role in the pathogenesis and perpetuation of $\mathrm{AF}[24,25]$. Indices of oxidative stress are increased with severity of kidney disease and correlates significantly with level of renal function [26]. For example, in the left atrium, expressions of nicotinamide adenine dinucleotide phosphate oxidase and malondialdehyde were increased by CKD [27].

\section{Limitations of the study}

Meta-analyses of observational studies present particular challenges because of inherent biases and differences in study designs. This meta- 
Table 2. Baseline data of patients included in the meta-analysis.

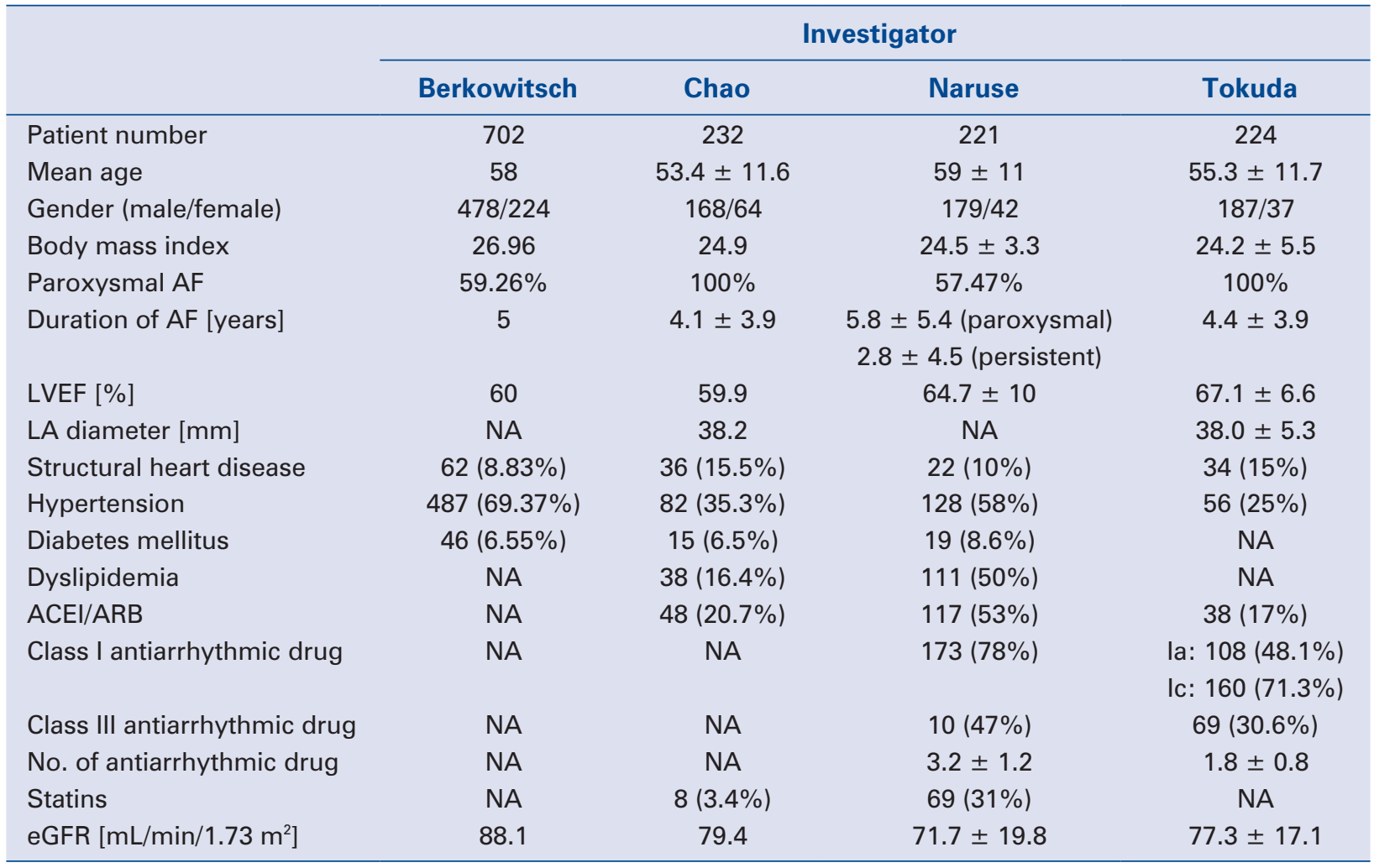

ACEI - angiotensin converting enzyme inhibitor; ARB — angiotensin receptor blocker; AF — atrial fibrillation; eGFR - estimated glomerular filtration rate; LA — left atrium; LVEF — left ventricular ejection fraction; NA — not applicable

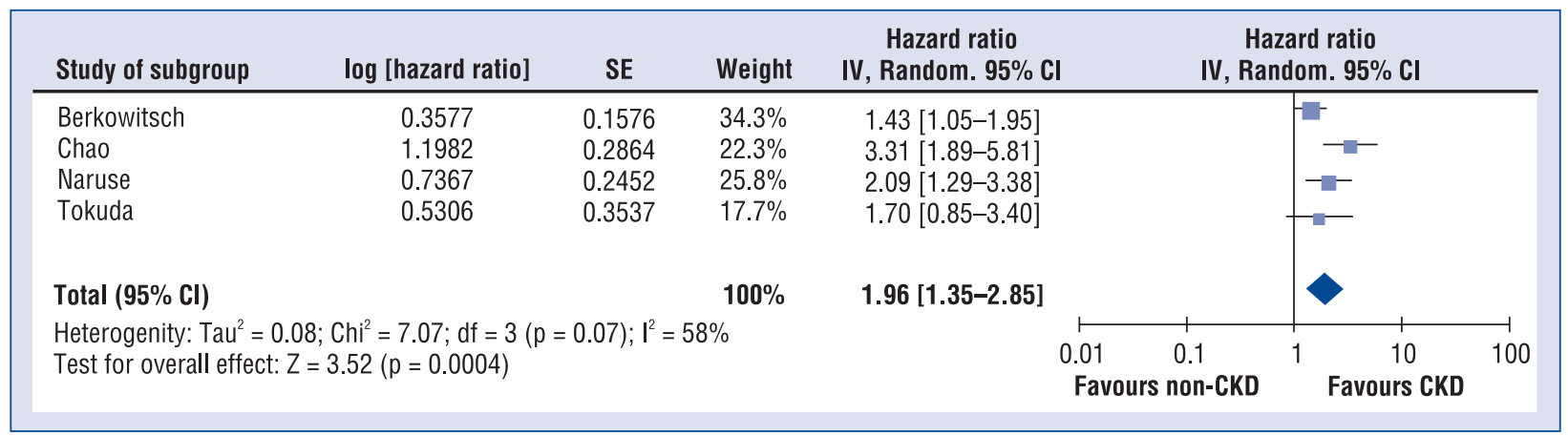

Figure 2. Comparison of atrial fibrillation recurrence following single catheter ablation between patients with and without chronic kidney disease (CKD); $\mathrm{Cl}$ - confidence interval; SE - standard error.

-analysis is limited because number of included studies is low and sample size is small. The results of all 4 studies are positive which may indicate a significant publication bias. Moreover, the present study was a meta-analysis of observational studies only. Because of the nature of the hypothesis, it is only possible for studies to be designed in this manner. Therefore, our analysis has demonstrated an association but not causality and is hypothesis generating. We used eGFR $<60 \mathrm{~mL} / \mathrm{min} / 1.73 \mathrm{~m}^{2}$ as a diagnostic standard for $\mathrm{CKD}$, but this is not the reference standard for diagnosing $\mathrm{CKD}$ and has its limitations. However, this did not alter the overall result. The study by Tokuda et al. [9] only gave univariate $\mathrm{HR}$ value and we manually calculated HR value of the study by Chao et al. [5]. Similar to other meta-analyses, because of the lack of data from the included studies, we were unable to adjust risk factors by multivariate regression. The study by Berkowitsch et al. [10] enrolled the largest 


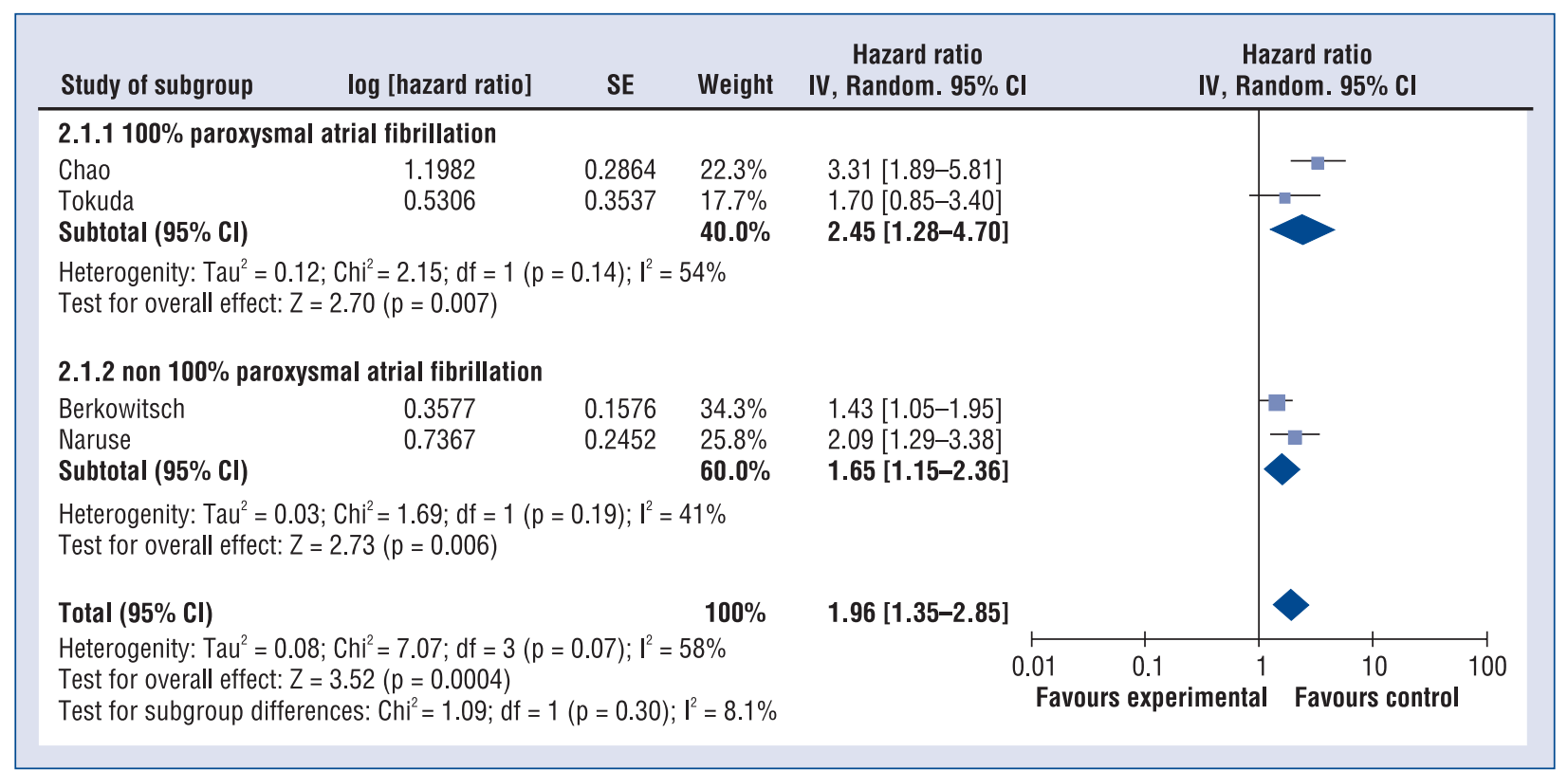

Figure 3. Subgroup analysis regarding different study population; SE — standard error; $\mathrm{Cl}$ - confidence interval.

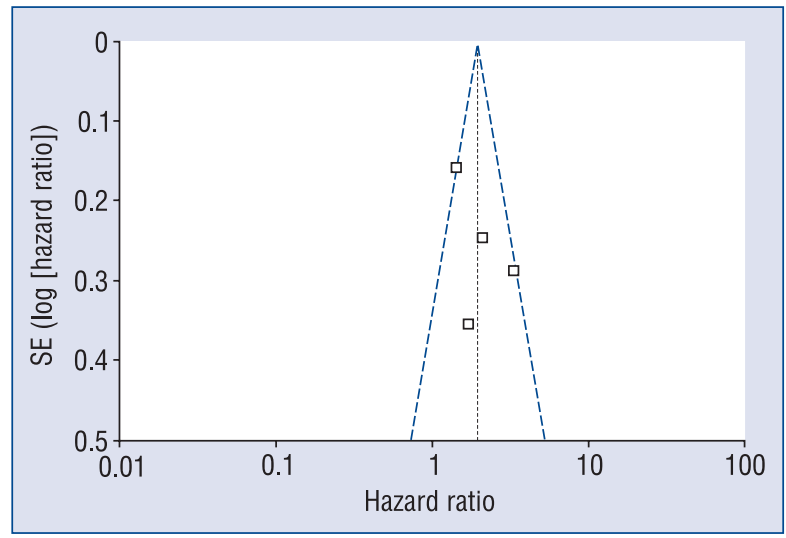

Figure 4. Funnel plot of the meta-analysis; SE — standard error.

number of patients, with a weight of $34.3 \%$ in our pooled result. It had the greatest influence on our findings. Therefore, our meta-analysis was subject to that study's inherent limitations.

\section{Conclusions}

In conclusion, $\mathrm{CKD}$ was associated with higher $\mathrm{AF}$ recurrence rate after single catheter ablation. Given that the CKD prevalence is rapidly increasing, there is an imperative need for better risk stratification of catheter ablation candidates.

Conflicts of interest: none declared

\section{References}

1. Takahashi Y, Takahashi A, Kuwahara T et al. Renal function after catheter ablation of atrial fibrillation. Circulation, 2011; 124: 2380-2387.

2. Bohnen M, Stevenson WG, Tedrow UB et al. Incidence and predictors of major complications from contemporary catheter ablation to treat cardiac arrhythmias. Heart Rhythm, 2011; 8: 1661-1666.

3. Kocheril AG. Arrhythmia issues in patients with renal disease. Semin Nephrol, 2001; 21: 57-65.

4. Naruse Y, Tada H, Sekiguchi Y et al. Concomitant chronic kidney disease increases the recurrence of atrial fibrillation after catheter ablation of atrial fibrillation: A mid-term follow-up. Heart Rhythm, 2011; 8: 335-341.

5. Chao TF, Lin YJ, Chang SL et al. Associations between renal function, atrial substrate properties and outcome of catheter ablation in patients with paroxysmal atrial fibrillation. Circ J, 2011; 75: 2326-2332.

6. Stroup DF, Berlin JA, Morton SC et al. Meta-analysis of observational studies in epidemiology: a proposal for reporting. Meta-analysis Of Observational Studies in Epidemiology (MOOSE) group. JAMA, 2000; 283: 2008-2012.

7. Harris RP, Helfand M, Woolf SH et al. Current methods of the US Preventive Services Task Force: a review of the process. Am J Prev Med, 2001; 20 (3 Suppl.): 21-35.

8. Levine M, Walter S, Lee H, Haines T, Holbrook A, Moyer V. Users' guides to the medical literature. IV. How to use an article about harm. Evidence-Based Medicine Working Group. JAMA, 1994; 271: 1615-1619.

9. Tokuda M, Yamane T, Matsuo S et al. Relationship between renal function and the risk of recurrent atrial fibrillation following catheter ablation. Heart, 2011; 97: 137-142.

10. Berkowitsch A, Kuniss M, Greiss $\mathrm{H}$ et al. Impact of impaired renal function and metabolic syndrome on the recurrence of atrial 
fibrillation after catheter ablation: a long term follow-up. Pacing Clin Electrophysiol, 2012; 35: 532-543.

11. Higgins JP, Thompson SG, Deeks JJ, Altman DG. Measuring inconsistency in meta-analyses. BMJ, 2003; 327: 557-560.

12. Geidel S, Lass M, Krause K et al. Persistent atrial fibrillation ablation concomitant to coronary surgery. Thorac Cardiovasc Surg, 2011; 59: 207-212.

13. Geidel S, Krause K, Boczor S et al. Ablation surgery in patients with persistent atrial fibrillation: An 8-year clinical experience. J Thorac Cardiovasc Surg, 2011; 141: 377-382.

14. Attaran S, Saleh HZ, Shaw M, Ward A, Pullan M, Fabri BM. Does the outcome improve after radiofrequency ablation for atrial fibrillation in patients undergoing cardiac surgery? A propensity-matched comparison. Eur J Cardiothorac Surg, 2012; 41: 806-810.

15. Bortone A, Boveda S, Pasquie JL et al. Sinus rhythm restoration by catheter ablation in patients with long-lasting atrial fibrillation and congestive heart failure: Impact of the left ventricular ejection fraction improvement on the implantable cardioverter defibrillator insertion indication. Europace, 2009; 11: 1018-1023.

16. Levey AS, Bosch JP, Lewis JB, Greene T, Rogers N, Roth D. A more accurate method to estimate glomerular filtration rate from serum creatinine: A new prediction equation. Modification of Diet in Renal Disease Study Group. Ann Intern Med, 1999; 130: 461-470.

17. Szczech LA. Atrial fibrillation: The beat is faster than the answers. Kidney Int, 2012; 81: 432-433.

18. Sandhu RK, Kurth T, Conen D, Cook NR, Ridker PM, Albert CM. Relation of renal function to risk for incident atrial fibrillation in women. Am J Cardiol, 2012; 109: 538-542.
19. House AA, Ronco C. The burden of cardiovascular risk in chronic kidney disease and dialysis patients (cardiorenal syndrome type 4). Contrib Nephrol, 2011; 171: 50-56.

20. Baber U, Howard VJ, Halperin JL et al. Association of chronic kidney disease with atrial fibrillation among adults in the United States: REasons for Geographic and Racial Differences in Stroke (REGARDS) Study. Circ Arrhythm Electrophysiol, 2011; 4: 26-32.

21. Alonso A, Lopez FL, Matsushita K et al. Chronic kidney disease is associated with the incidence of atrial fibrillation: The Atherosclerosis Risk in Communities (ARIC) study. Circulation, 2011; 123: 2946-2953.

22. Klein RM, Vester EG, Brehm MU et al. Inflammation of the myocardium as an arrhythmia trigger. Z Kardiol, 2000; 89 (Suppl. 3): 24-35.

23. Voroneanu L, Covic A. Arrhythmias in hemodialysis patients. J Nephrol, 2009; 22: 716-725.

24. Liu T, Li GP, Li LJ, Korantzopoulos P. Association between C-reactive protein and recurrence of atrial fibrillation after successful cardioversion: A meta-analysis. J Am Coll Cardiol, 2007; 49: 1642-1648.

25 Liu T, Li L, Korantzopoulos P, Goudevenos JA, Li G. Meta-analysis of association between C-reactive protein and immediate success of electrical cardioversion in persistent atrial fibrillation. Am J Cardiol, 2008; 101: 1749-1752.

26. Dounousi E, Papavasiliou E, Makedou A et al. Oxidative stress is progressively enhanced with advancing stages of CKD. Am J Kidney Dis, 2006; 48: 752-760.

27. Fukunaga N, Takahashi N, Hagiwara S et al. Establishment of a model of atrial fibrillation associated with chronic kidney disease in rats and the role of oxidative stress. Heart Rhythm, 2012; 9: 2023-2031. 